\title{
BMJ Open Risks of postextraction bleeding after receiving direct oral anticoagulants or warfarin: a retrospective cohort study
}

\author{
Takahiro Yagyuu, ${ }^{1}$ Mao Kawakami, ${ }^{1}$ Yoshihiro Ueyama, ${ }^{1}$ Mitsuhiko Imada, ${ }^{1}$ \\ Miyako Kurihara, ${ }^{1}$ Yumiko Matsusue, ${ }^{1}$ Yuichiro Imai, ${ }^{1,2}$ Kazuhiko Yamamoto, ${ }^{1}$ \\ Tadaaki Kirita $^{1}$
}

\begin{abstract}
To cite: Yagyuu T, Kawakami M, Ueyama Y, et al. Risks of postextraction bleeding after receiving direct oral anticoagulants or warfarin: a retrospective cohort study. BMJ Open 2017;7:e015952. doi:10.1136/ bmjopen-2017-015952

- Prepublication history and additional material are available. To view these files please visit the journal online (http://dx.doi. org/10.1136/bmjopen-2017015952).
\end{abstract}

Received 27 March 2017 Revised 30 May 2017

Accepted 5 June 2017

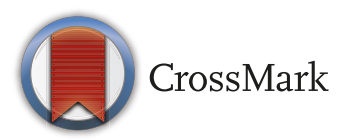

${ }^{1}$ Department of Oral and Maxillofacial Surgery, Nara Medical University, Nara, Japan ${ }^{2}$ Department of Oral and Maxillofacial Surgery,

Rakuwakai Otowa Hospital, Kyoto, Japan

Correspondence to Takahiro Yagyuu;

t-yagyuu@naramed-u.ac.jp

\section{ABSTRACT}

Objective The effect of direct oral anticoagulants (DOACs) on the risk of bleeding after tooth extraction remains unclear. This study aimed to evaluate the incidence of postextraction bleeding among patients who received DOAC and vitamin $\mathrm{K}$ antagonists (VKAs), such as warfarin. Design This study was a retrospective cohort analysis. Incidence rates and propensity score-matched regression models were used to compare the risks of bleeding after tooth extractions involving DOACs and VKAs.

Setting The study took place in a single university hospital in Japan.

Participants Between April 2013 and April 2015, 543 patients underwent a total of 1196 simple tooth extractions.

Primary outcome measure The primary outcome measure was the occurrence of postextraction bleeding, which was defined as bleeding that could not be stopped by biting down on gauze and required medical treatment between $30 \mathrm{~min}$ and 7 days after the extraction.

Results A total of 1196 tooth extractions (634 procedures) in 541 patients fulfilled the study criteria, with 72 extractions (41 procedures) involving DOACs, 100 extractions (50 procedures) involving VKAs and 1024 extractions (543 procedures) involving no anticoagulants. The incidences of postextraction bleeding per tooth for the DOAC, VKA and no anticoagulant extractions were $10.4 \%, 12.0 \%$ and $0.9 \%$, respectively. The incidences of postextraction bleeding per procedure for DOACs, VKAs and no anticoagulants were $9.7 \%, 10.0 \%$ and $1.1 \%$, respectively. In comparison to the VKA extractions, the DOAC extractions did not significantly increase the risk of postextraction bleeding (OR 0.69, 95\% Cls 0.24 to 1.97 ; $\mathrm{p}=0.49$ ).

Conclusions The risk of postextraction bleeding was similar for DOAC and VKA extractions.

\section{INTRODUCTION}

Anticoagulation therapy is recommended to prevent strokes and systemic embolisms in patients with atrial fibrillation, ${ }^{1}$ thromboembolisms in patients with mechanical heart valves ${ }^{2}$ and deep vein thrombosis in patients undergoing knee or hip replacement surgery. ${ }^{3}$ Vitamin K antagonists (VKAs), such

\section{Strengths and limitations of this study}

- This is one of the first studies to evaluate the incidence of postextraction bleeding among patients who received DOACs.

- This was a small-scale, retrospective study in which data were obtained from a single facility.

- Since the number of patients and bleeding severity scores were not evenly distributed, the results cannot be representative of patients with a high risk of bleeding.

as warfarin, have historically been the only available oral anticoagulants, despite their narrow therapeutic index, requirement for monitoring and numerous drug-drug and food interactions. ${ }^{1}$ Therefore, the recent introduction of direct oral anticoagulants (DOACs) has provided therapeutic options with several practical advantages, such as fewer interactions and no need to perform routine blood monitoring. There are four types of DOACs that have been approved in the USA, Japan and several European countries: dabigatran (a direct thrombin inhibitor) and rivaroxaban, apixaban and edoxaban (factor Xa inhibitors).

In 2015, the US Food and Drug Administration approved idarucizumab as a reversal agent for dabigatran. ${ }^{4}$ However, there are no known agents for reversing bleeding in patients receiving rivaroxaban, apixaban or edoxaban. Furthermore, in the clinical setting, the magnitude of the bleeding risk that is associated with DOACs remains unclear. A recent meta-analysis of 71684 patients revealed a $25 \%$ increase in gastrointestinal bleeding among patients who received DOACs, as compared with patients who received warfarin. ${ }^{5}$ A recent cohort study of 219027 patients who received anticoagulant therapy reported a similar increase of gastrointestinal bleeding among patients who 
received DOACs, as compared with patients who received VKAs. ${ }^{6}$ However, few studies have evaluated the risk and incidence of postextraction bleeding among patients who receive DOACs. ${ }^{7-9}$ Thus, an index for assessing the risk of bleeding among patients who receive DOACs would be clinically useful.

Various bleeding risk scores have been proposed to evaluate major bleeding risks among patients who receive anticoagulants, such as the Hypertension, Abnormal Renal/Liver Function, Stroke, Bleeding History or Predisposition, Labile International Normalised Ratio (INR), Elderly, Drugs/Alcohol Concomitantly (HAS-BLED) score ${ }^{10}$ the Anticoagulation and Risk Factors in Atrial Fibrillation (ATRIA) score ${ }^{11}$ and the Outcomes Registry for Better Informed Treatment (ORBIT) score. ${ }^{12}$ In particular, the HAS-BLED score has gained popularity for use among patients with atrial fibrillation who receive VKAs because the HAS-BLED score is significantly associated with both major and non-major bleeding risks (HRs (95\% CI) 2.4 (1.28 to 4.52 ) and 1.85 (1.43 to 2.40 ), respectively). ${ }^{13} 14$ However, it is unclear whether these scores can predict the risk of postextraction bleeding among patients who receive anticoagulants. Therefore, the present study aimed to retrospectively evaluate the incidence of postextraction bleeding among patients who receive DOACs or VKAs and to quantify the abilities of the HAS-BLED, ATRIA and ORBIT scores to predict postextraction bleeding.

\section{METHODS}

\section{Patients and design}

This study's retrospective design was approved by the ethics committee of Nara Medical University (approval date: 19 October 2015; approval number: 197), and the study was performed in accordance with the Declaration of Helsinki guidelines. Medical records were used to identify patients who underwent simple tooth extraction(s) between April 2013 and April 2015 within the department of oral and maxillofacial surgery at Nara Medical University Hospital. A simple tooth extraction was defined as tooth extraction without removing the surrounding bone or cutting the gum. Patients were excluded if they required extractions of deciduous teeth. Based on medical record data, extractions were categorised according to whether the patient received DOACs, VKAs or no anticoagulants (the control group) during the procedure. Prior to the extractions, all patients underwent blood tests within 1 month of the surgery. These tests included complete blood counts, platelet count, activated partial thromboplastin time (APTT), prothrombin time-international normalised ratio (PT-INR) and haemoglobin levels, total bilirubin, aspartate aminotransferase, alanine aminotransferase, alkaline phosphatase, blood urea nitrogen and creatinine. In addition, on the day of the tooth extraction, patients who were to receive VKAs had their PT-INR measured. According to the 'Guidelines for Patients on Antithrombotic Therapy Requiring Dental
Extraction, 15 and the 'Guidelines for Pharmacotherapy of Atrial Fibrillation', ${ }^{16}$ if the PT-INR was $>3$, the extraction was postponed and the patient's physician was consulted.

\section{Study variables}

The primary outcome measure was the occurrence of postextraction bleeding, which was defined as bleeding that could not be stopped by biting down on gauze and that required medical treatment between $30 \mathrm{~min}$ and 7 days after the tooth extraction. The study variables were age, sex, PT-INR, APTT, local haemostatic agents (eg, absorbable oxidised cellulose or gelatine sponge), wound suturing and the prescription of non-steroidal anti-inflammatory drugs after the extraction. For the extractions that involved anticoagulants, the HAS-BLED, ATRIA and ORBIT scores were calculated. The HAS-BLED score assigns points for the presence of each of the following risk factors: hypertension (uncontrolled systolic blood pressure of $>160 \mathrm{~mm} \mathrm{Hg}$ ), abnormal renal and/or liver function, previous stroke, bleeding history or predisposition, labile PT-INR, $\geq 65$ years of age and concomitant drugs and/or excessive alcohol consumption. ${ }^{10}$ In the present study, labile PT-INR was defined as $<60 \%$ time in the therapeutic range, which was calculated based on the patient's last three PT-INR measurements taken during the 3 months prior to the extraction. In patients who received DOACs, 0 points were assigned for labile PT-INRs. The ATRIA score was calculated using the following parameters: anaemia (haemoglobin levels of $<13 \mathrm{~g} / \mathrm{dL}$ in men and $<12 \mathrm{~g} / \mathrm{dL}$ in women; three points), severe renal disease (estimated glomerular filtration rate of $<30 \mathrm{~mL} / \mathrm{min} / 1.73 \mathrm{~m}^{2}$; three points), $\geq 75$ years of age (two points), prior bleeding and hypertension. ${ }^{11}$ The ORBIT score was calculated using the following parameters: one point for $>74$ years of age, insufficient kidney function (estimated glomerular filtration rate of $<60 \mathrm{~mL}$ / $\min / 1.73 \mathrm{~m}^{2}$ ) and/or antiplatelet treatment and two points for a clinical history of bleeding and/or the presence of anaemia or abnormal haemoglobin levels ( $<13 \mathrm{mg} / \mathrm{dL}$ for men and $<12 \mathrm{mg} / \mathrm{dL}$ for women $){ }^{12}$

\section{Statistical analysis}

Based on the methods of previous studies, ${ }^{17}$ data for each extracted tooth were collected, and patients were counted multiple times if they underwent multiple extractions.

The incidence of postextraction bleeding was calculated for the extractions that involved DOACs, VKAs or no anticoagulants. A multivariate logistic regression analysis was performed to identify risk factors for postextraction bleeding, and explanatory variables with a significance level of $\mathrm{p}<0.05$ in the univariate analyses were included in the multivariate model. One-to-one propensity score matching was used to adjust for differences in the baseline covariates (age, sex, antiplatelet treatment, HAS-BLED score, local haemostatic agents, wound suturing and prescription of non-steroidal anti-inflammatory drugs) during the analyses that compared the risks of postextraction bleeding in the DOAC and VKA 
groups. Continuous variables were compared using the paired t-test, and dichotomous variables were compared using McNemar's test. Explanatory variables with a significance level of $p<0.05$ in the univariate propensity score analyses were included in the multivariate logistic regression model to evaluate the postextraction bleeding risks in the DOAC and VKA groups. To compare the abilities of the HAS-BLED, ATRIA and ORBIT scores to predict postextraction bleeding in cases that involved anticoagulants, each score's sensitivity, specificity and positive or negative likelihood ratios (LR+ and LR-, respectively) were calculated.

Receiver operating characteristic (ROC) curves were plotted for the three scores, and the areas under the curves (AUCs) were used to determine the cut-off values. The AUCs were compared using the De Long and ClarkePearson method, ${ }^{18}$ and logistic regression analysis was used to investigate the association between the three bleeding risk scores and postextraction bleeding. All data were analysed using STATA software V.13.0 (StataCorp, College Station, Texas, USA).

\section{RESULTS}

\section{Case characteristics}

The characteristics of the included cases are summarised in online supplementary appendix table 1 . A total of 1196 tooth extractions (634 procedures) in 541 patients fulfilled the inclusion criteria, with 72 extractions $(6.0 \%)$ involving DOACs (four extractions also involved antiplatelet agents), 100 extractions $(8.3 \%)$ involving VKAs (15 extractions also involved antiplatelet agents) and 1024 extractions $(85.6 \%)$ involving no anticoagulants (191 extractions also involved antiplatelet agents). The 72 DOAC extractions included 31 extractions with apixaban, 24 extractions with rivaroxaban, 14 extractions with dabigatran and three extractions with edoxaban. For all patients, antithrombotic agents were not interrupted during the extraction. The mean PT-INRs for the DOAC and VKA extractions were 1.17 (95\% CI 1.13 to 1.21) and 1.69 (95\% CI 1.61 to 1.77), respectively. The mean APTTs for the DOAC and VKA extractions were $33.0(95 \%$ CI 31.5 to 34.4 ) and 33.6 (95\% CI 32.4 to 34.8 ), respectively. All patients exhibited PT-INRs of $<3$ and APTTs that were less than twofold above the normal range's upper limit. There were no cases of postponed extractions. Postextraction bleeding events were observed in 29 of the 1196 extractions (2.4\%), 15 of the 634 procedures $(2.3 \%)$ and 15 of the 541 patients $(2.7 \%)$. Of the 29 extractions with postextraction bleeding, seven involved DOACs, 12 involved VKAs and 10 involved no anticoagulants. The incidence of postextraction bleeding for the DOAC, VKA and no anticoagulant extractions was $10.4 \%$, $12.0 \%$ and $0.9 \%$, respectively. When we analysed the data per procedure, the incidence for DOACs, VKAs and no anticoagulants was $9.7 \%, 10.0 \%$ and $1.1 \%$, respectively (table 1). All bleeding could be stopped by local haemostatic treatment, including compression with gauze,

\begin{tabular}{|c|c|c|}
\hline & Per tooth & Per procedure \\
\hline \multicolumn{3}{|l|}{ DOACs } \\
\hline Events/total (incidence) & $7 / 72(10.4 \%)$ & $4 / 41(9.7 \%)$ \\
\hline \multicolumn{3}{|l|}{ VKAs } \\
\hline Events/total (incidence) & 12/100 (12.0\%) & $5 / 50(10.0 \%)$ \\
\hline \multicolumn{3}{|l|}{ No anticoagulants } \\
\hline Events/total (incidence) & 10/1,024 (0.9\%) & $6 / 543(1.1 \%)$ \\
\hline
\end{tabular}

DOACs, direct oral anticoagulants; VKAs, vitamin K antagonists.

injection of local anaesthetics with vasoconstrictor and/ or use of absorbable haemostats. No general complications were noted.

\section{Risk factors for postextraction bleeding}

Univariate analysis revealed that postextraction bleeding was associated with older age (OR 1.04; $\mathrm{p}=0.003$ ), receiving DOACs (OR 5.39; $<<0.001$ ), receiving VKAs $($ OR $8.65 ; \mathrm{p}<0.001)$ and use of local haemostatic agents (OR $4.41 ; \mathrm{p}<0.001)$. Use of antiplatelet agents was not a risk factor for postextraction bleeding $(\mathrm{p}=0.34)$. Multivariate logistic regression analysis revealed that postextraction bleeding was independently associated with receiving DOACs $($ OR 8.69; $\mathrm{p}<0.001)$ and receiving VKAs (OR 8.88; $\mathrm{p}<0.001$ ) (table 2).

\section{Risks of postextraction bleeding for DOACs and VKAs}

Analysis matched $93.0 \%$ of the DOAC extractions to $67.0 \%$ of the VKA extractions, which created 67 matched pairs. These pairs exhibited similar baseline clinical characteristics (table 3). The mean PT-INRs for the DOAC and VKA extractions were $1.15 \pm 0.12$ and $1.63 \pm 0.39$, respectively. Compared with the VKA extractions, the DOAC extractions did not have a significantly increased risk of postextraction bleeding (OR $0.69,95 \%$ CI 0.24 to 1.97, $\mathrm{p}=0.49$ ).

\section{Ability of the bleeding risk scores' to predict postextraction bleeding in the anticoagulant group}

The median HAS-BLED score in the anticoagulant group (DOAC and VKA extractions) was 2 (IQR 1-2), the median ATRIA score was 2 (IQR 0-3) and the median ORBIT score was 3 (IQR 1-4) (see online supplementary appendix table 2). The AUCs for the HAS-BLED, ATRIA and ORBIT scores were $0.65,0.74$ and 0.70 , respectively (figure 1). Multivariate logistic regression analysis revealed that the HAS-BLED, ATRIA and ORBIT scores were significantly associated with the risk of postextraction bleeding (OR (95\% CIs) 2.93 (1.05 to 8.12), 9.69 (3.34 to 28.1) and 2.91 ( 1.10 to 7.71 ); $\mathrm{p}=0.03, \mathrm{p}<0.001$ and $\mathrm{p}=0.03$, respectively). Based on the ROC analysis, the optimal cut-off values for the HAS-BLED, ATRIA and ORBIT scores were 2, 5 and 4, respectively. The sensitivity, specificity and LR+ of the HAS-BLED score were $68.4 \%, 57.5 \%$ and 1.61 , respectively. The sensitivity, specificity and LR+ of the ATRIA score were $47.3 \%, 91.5 \%$ and 
Table 2 Risk factors for postextraction bleeding

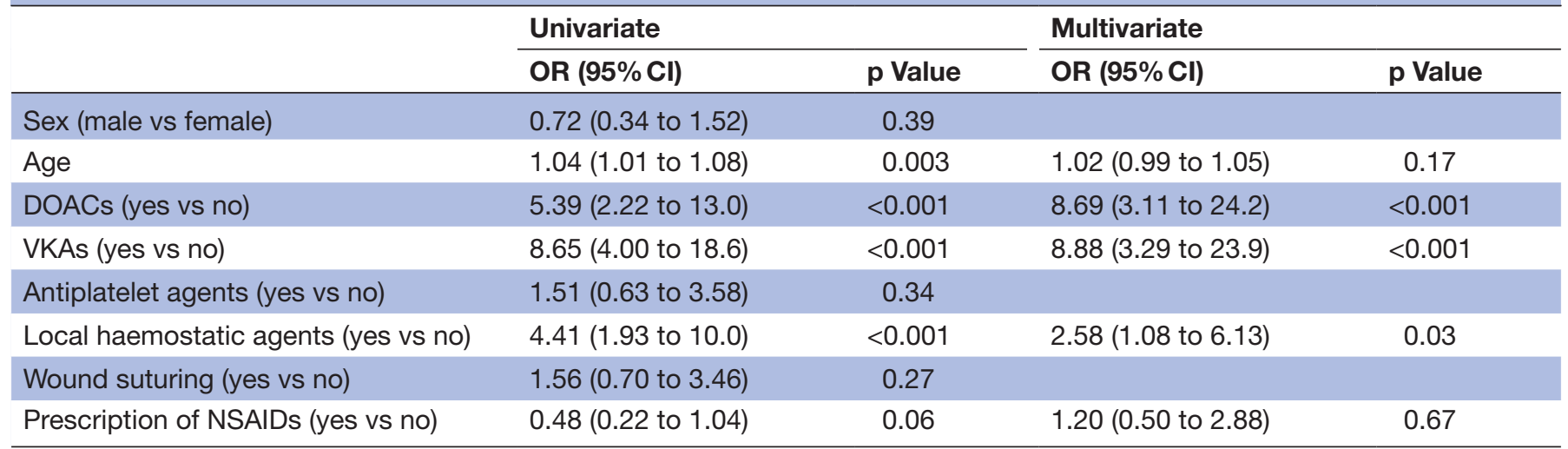

DOACs, direct oral anticoagulant; NSAIDs, non-steroidal anti-inflammatory drugs; VKAs, vitamin K antagonists.

5.57, respectively. The sensitivity, specificity and LR+ of the ORBIT score were $57.8 \%, 67.9 \%$ and 1.80 , respectively (table 4).

\section{Comparing the AUCs for the HAS-BLED, ATRIA and ORBIT} scores

Based on the AUC analyses, the HAS-BLED, ATRIA and ORBIT scores exhibited similar abilities to predict postextraction bleeding (HAS-BLED vs ATRIA, $p=0.12$; HAS-BLED vs ORBIT, $\mathrm{p}=0.27$; and ATRIA vs ORBIT, $\mathrm{p}=0.28)$.

\section{DISCUSSION}

Simple dental procedures without VKA cessation have become standard among patients who receive VKAs. ${ }^{19} 20$ However, there is no consensus regarding whether DOACs should be discontinued during dental procedures. Thus, given the challenges of monitoring and reversing the anticoagulation effects of DOACs, dental and medical professionals should carefully consider the perioperative management of patients who receive DOACs, as their care may differ from that of patients who receive VKAs.

Table 3 Characteristics of the propensity score-matched cohort for DOAC and VKA extractions

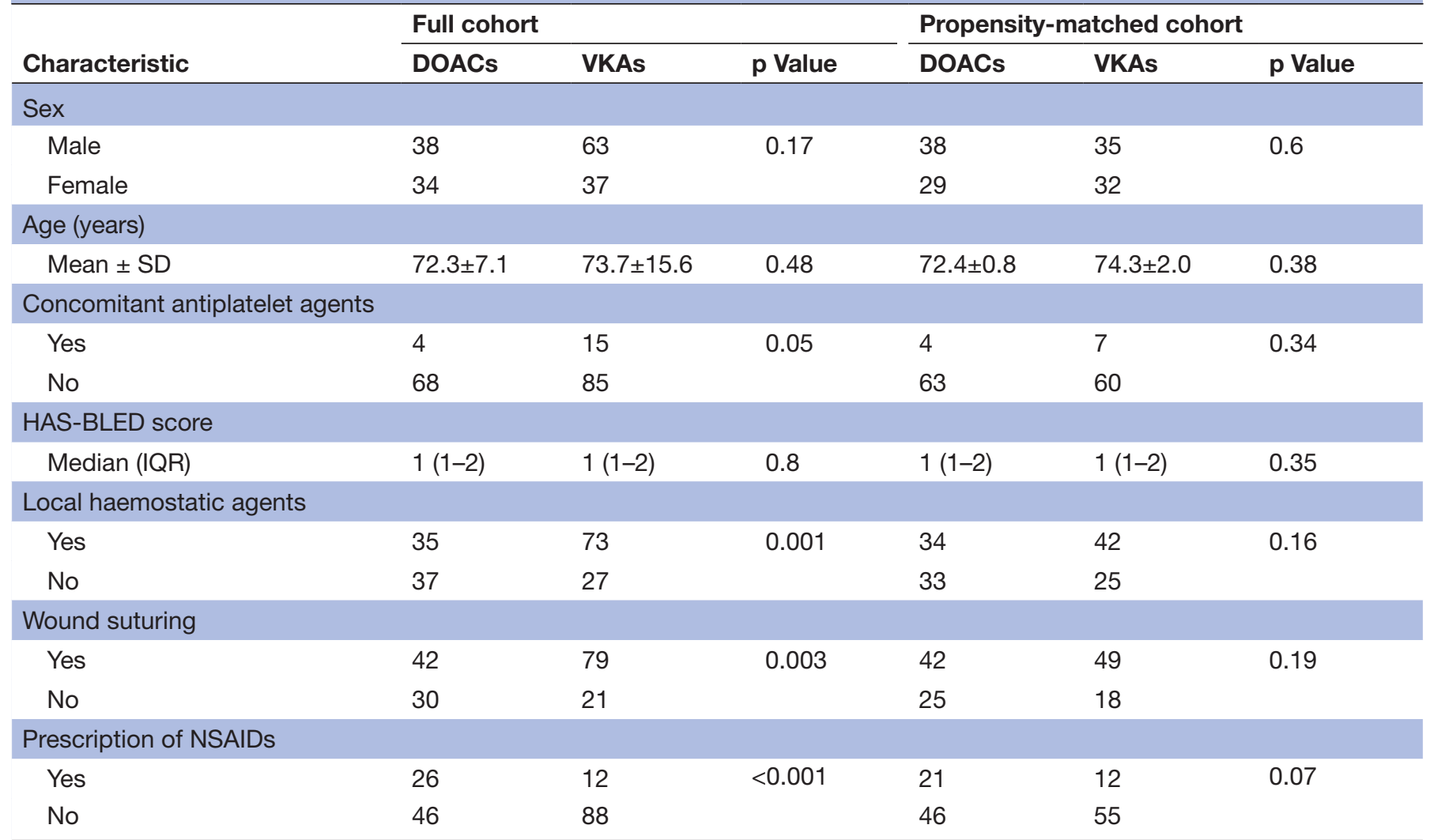

DOACs, direct oral anticoagulants; HAS-BLED, Hypertension, Abnormal renal/liver function, Stroke, Bleeding history or predisposition, Labile international normalised Ratio, Elderly, Drugs/alcohol; NSAIDs, non-steroidal anti-inflammatory drugs; VKAs, vitamin K antagonists. 


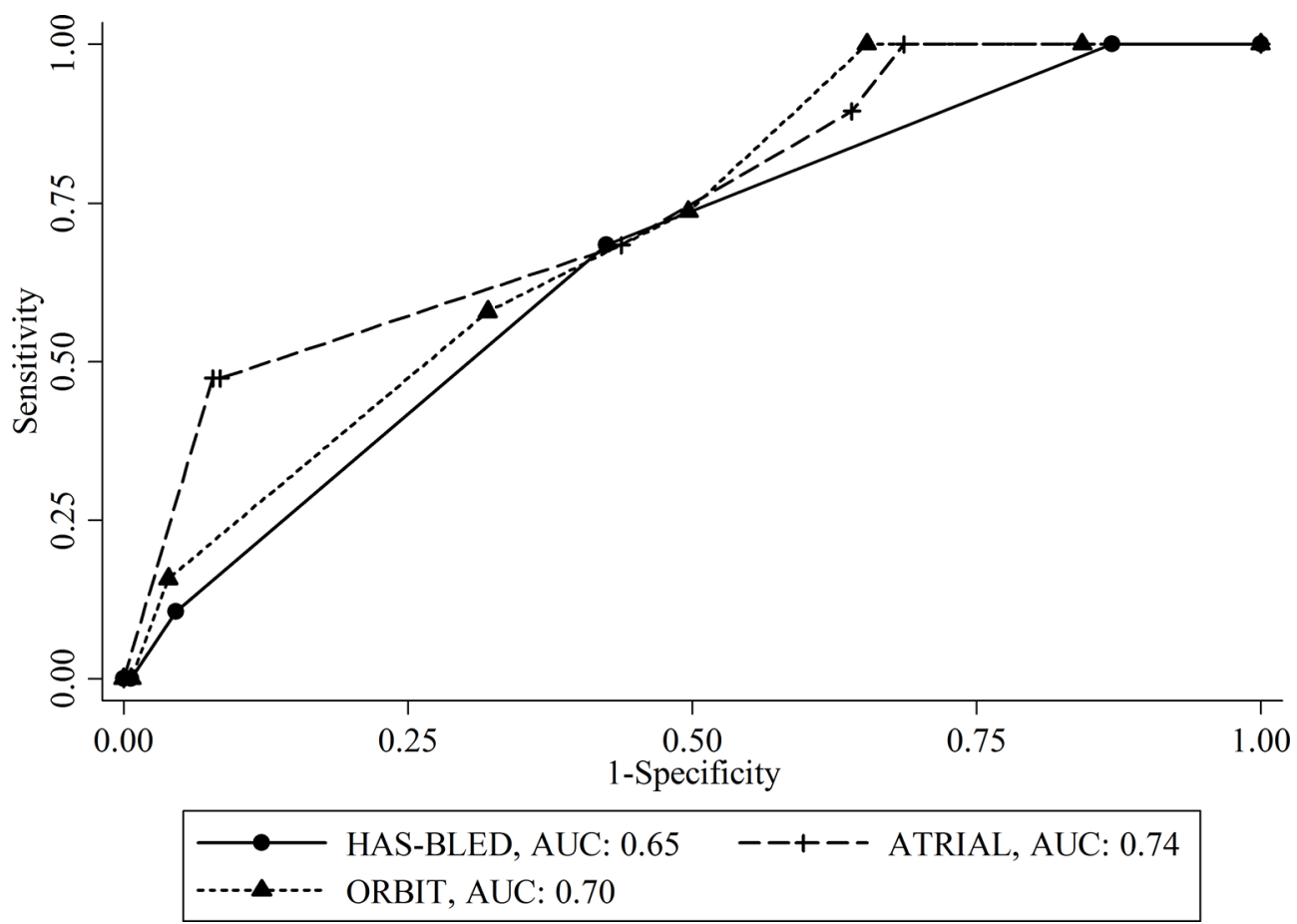

Figure 1 Receiver operating characteristic curves and areas under the curves (AUCs) for the three bleeding risk scores. The AUC for the Hypertension, Abnormal renal/liver function, Stroke, Bleeding history or predisposition, Labile international normalised Ratio, Elderly, Drugs/alcohol (HAS-BLED) score was 0.65, the AUC for the Anticoagulation and Risk Factors in Atrial Fibrillation (ATRIA) score was 0.74 and the AUC for the Outcomes Registry for Better Informed Treatment (ORBIT) score was 0.70 .

Several perioperative approaches have been recommended, such as continuing DOAC, delaying invasive treatment as late as possible after the last DOAC dose or discontinuing DOACs for 24-48hours. Although cases of limited dental surgery may benefit from the first two more conservative perioperative options, ${ }^{3} 4^{20-23}$ few studies have evaluated DOAC-associated risks of postextraction bleeding.

In the Department of Oral and Maxillofacial Surgery at Nara Medical University Hospital, DOAC administration during simple tooth extractions in patients with no significant abnormalities in the presurgery blood tests is not typically interrupted. However, according to the 'Guidelines for Patients on Antithrombotic Therapy Requiring Dental Extraction' 15 and the "Guidelines for Pharmacotherapy of Atrial Fibrillation', ${ }^{16}$ if a patient is receiving a VKA and has a PT-INR of $>3$, the extraction is postponed, and the patient's physician is consulted. Yet, all of the extractions in the present study were performed without antithrombotic agent discontinuation. The incidence rates of postextraction bleeding among extractions that involved DOACs, VKAs or no anticoagulants were $10.4 \%, 12.0 \%$ and $0.9 \%$, respectively, and receiving DOACs or VKAs was significant risk factors for postextraction bleeding (ORs 8.69 and 8.88, respectively). These findings are in accordance with reported incidences of postextraction bleeding among patients who did not cease anticoagulants during the extraction, which range from $0 \%$ to $26 \% .^{91724-35}$ The discrepancy among the incidences may be related to the use of different definitions for postextraction bleeding. Multivariate logistic regression analysis also revealed that postextraction bleeding was independently associated with local haemostatic agents (OR 2.58), but this observation is believed to be a

Table 4 The three bleeding risk scores' abilities to predict postextraction bleeding in the anticoagulant group

\begin{tabular}{llllll} 
& $\begin{array}{l}\text { Sensitivity } \\
\text { (cut-off) }\end{array}$ & $\begin{array}{l}\text { Specificity } \\
\text { (cut-off) }\end{array}$ & $\begin{array}{l}\text { LR+ } \\
\text { (cut-off) }\end{array}$ & $\begin{array}{l}\text { LR- } \\
\text { (cut-off) }\end{array}$ & OR (95\% Cl); p Value \\
\hline HAS-BLED & $\begin{array}{l}68.4 \% \\
(\geq 2)\end{array}$ & $\begin{array}{l}57.5 \% \\
(\geq 2)\end{array}$ & $\begin{array}{l}1.61 \\
(\geq 2)\end{array}$ & $\begin{array}{l}0.54 \\
(\geq 2)\end{array}$ & $2.93(1.05$ to 8.12); 0.03 \\
ATRIA & $47.3 \%$ & $91.5 \%$ & 5.57 & 0.57 & $9.69(3.34$ to 28.1);<0.001 \\
& $(\geq 5)$ & $(\geq 5)$ & $(\geq 5)$ & $(\geq 5)$ & $2.91(1.10$ to 7.71$) ; 0.03$ \\
ORBIT & $57.8 \%$ & $67.9 \%$ & 1.80 & 0.61 & $(\geq 4)$ \\
& $(\geq 4)$ & $(\geq 4)$ & $(\geq 4)$ & & \\
\hline
\end{tabular}

LR+, positive likelihood ratios; LR-, negative likelihood ratios. 
response to excessive bleeding during extraction, not an actual cause of bleeding.

To compare the effects of DOACs and VKAs on the risk of postextraction bleeding, one-to-one propensity score matching of extractions that involved DOACs or VKAs was conducted. The risks were found to be similar for both groups, and this finding is consistent with the finding of a previous study that evaluated the risk of gastrointestinal bleeding among patients who received dabigatran and rivaroxaban or warfarin. ${ }^{6}$ Thus, since simple dental procedures are routinely performed without VKA cessation, it may be appropriate to consider maintaining DOAC treatment during these procedures.

Multivariate logistic regression analysis revealed that the HAS-BLED, ATRIA and ORBIT scores were significantly associated with postextraction bleeding risk. Thus, the scores' ability to predict postextraction bleeding was also evaluated through ROC analyses. In these analyses, an AUC of 0.5 indicates that the test's performance is equal to random chance, an AUC of 0.5-0.7 indicates a low predictive ability, an AUC of 0.7-0.9 indicates a moderate predictive ability and an AUC of 0.9-1.0 indicates a high predictive ability. ${ }^{36-39}$ The AUCs for the HAS-BLED, ATRIA and ORBIT scores were 0.65, 0.74 and 0.70 , respectively, which indicates that their predictive abilities were low, moderate and moderate, respectively. These outcomes may be related to the fact that the HAS-BLED and ATRIA scores were developed for patients taking warfarin, ${ }^{10}{ }^{11}$ and the ORBIT score was developed from a cohort with few DOAC patients $(6.5 \%){ }^{12}$ However, these AUCs may be typical of clinical risk scores that are based on clinical features, including those that are used to predict the risk of major bleeding and stroke. In this context, the AUCs of the HAS-BLED, ATRIA and ORBIT scores for predicting major bleeding are $0.65,0.61$ and 0.61 , respectively. ${ }^{14}$ Although these scores were developed to estimate major bleeding risks, ${ }^{10-12}$ they may also be helpful for predicting postextraction bleeding before dental procedures. For normal evaluations, high risks of major bleeding are associated with a HAS-BLED score of $\geq 3$, an ATRIA score of $\geq 5$ and an ORBIT score of $\geq 4$. In the present study, postextraction bleeding was associated with a HAS-BLED score of $\geq 2$, an ATRIA score of $\geq 5$ and an ORBIT score of $\geq 4$.

The present study has several limitations. First, this was a small-scale, retrospective study, and the data were obtained from a single institution. Furthermore, on the day of the tooth extraction(s), none of the patients had a PT-INR of $>3$, and their mean PT-INR was $1.69(95 \%$ CI 1.61 to 1.77 ). This low PT-INR value may be related to the recommended PT-INR therapeutic range of 1.6-2.6 for elderly Japanese patients ( $\geq 70$ years of age), which is noticeably different from the recommended PT-INR range of 2.0-3.0 for Caucasian patients. ${ }^{40}$ Therefore, the participants of this study may not be representative of all patients who receive VKAs. Second, since the number of patients and bleeding severity scores were not evenly distributed, results cannot be representative of patients with a high risk of bleeding, and it may not be appropriate to extrapolate the results to high-risk patients who receive anticoagulants. In addition, the incidences of postextraction bleeding among the DOAC and VKA extractions may be lower than the incidences among the general population of patients who receive oral anticoagulants. Third, statistical analyses were performed per tooth extraction, and not per procedure, which increases the possibility of bias because patients could be counted multiple times. However, previous studies did not find a significant correlation between the number of extracted teeth and the incidence of postextraction bleeding. ${ }^{25} 27313541$ Furthermore, when we analysed the data per procedure, postextraction bleeding events were observed in 4 of 41 procedures $(9.7 \%), 5$ of 50 procedures $(10.0 \%)$ and 6 of 543 procedures $(1.1 \%)$ involving DOACs, VKAs and no anticoagulants, respectively. In the per tooth analysis, the postextraction incidences for DOACs, VKAs and no anticoagulants were $10.4 \%, 12.0 \%$ and $0.9 \%$, respectively. The postextraction incidences in the per-procedure analyses were similar to those in the per-tooth analyses. The mean number of teeth extracted per procedure was $2.16 \pm 1.69$ in the postextraction bleeding group and $1.83 \pm 1.33$ in the non-postextraction bleeding group. There was no significant association between postextraction bleeding and the number of teeth extracted per procedure $(\mathrm{p}=0.40)$. These data suggest that any bias from the per tooth analysis likely had minimal effect on the study's findings.

\section{CONCLUSION}

This retrospective cohort study is one of the first studies to evaluate the incidence of postextraction bleeding (10.4\%) among extractions that involved DOACs. Furthermore, the study found similar risks of postextraction bleeding among extractions that involved DOACs and extractions that involved VKAs. Finally, this study found that the HAS-BLED, ATRIA and ORBIT scores had low, moderate and moderate abilities, respectively, to predict postextraction bleeding among patients who received anticoagulants.

Contributors TY made substantial contributions to the study's design and drafting of the manuscript. MK, YU and MI made substantial contributions to the data acquisition and drafting of the manuscript. MK, YM, Yl and KY made substantial contributions to statistical analyses and drafting of the manuscript. TK conducted the study made substantial contributions to interpretation of data and revising of the manuscript.

Funding This research received no specific grant from any funding agency in thepublic, commercial or not-for-profit sectors.

Competing interests None declared.

Patient consent This study is retrospective chart review.

Ethics approval This study's retrospective design was approval by the ethics committee of Nara Medical University, Nara, Japan (approval date: 19 0ctober 2015; approval number: 197), and the study was performed in accordance with the Declaration of Helsinki.

Provenance and peer review Not commissioned; externally peer reviewed.

Data sharing statement Extra data can be accessed via the Dryad data repository at http://datadryad.org/ with the doi:10.5061/dryad.55jq5. 
Open Access This is an Open Access article distributed in accordance with the Creative Commons Attribution Non Commercial (CC BY-NC 4.0) license, which permits others to distribute, remix, adapt, build upon this work non-commercially, and license their derivative works on different terms, provided the original work is properly cited and the use is non-commercial. See: http://creativecommons.org/ licenses/by-nc/4.0/

(c) Article author(s) (or their employer(s) unless otherwise stated in the text of the article) 2017. All rights reserved. No commercial use is permitted unless otherwise expressly granted.

\section{REFERENCES}

1. Keeling D, Baglin T, Tait C, et al. Guidelines on oral anticoagulation with warfarin - fourth edition. Br J Haematol 2011;154:311-24.

2. Wigle $P$, Hein $B$, Bloomfield $\mathrm{HE}$, et al. Updated guidelines on outpatient anticoagulation. Am Fam Physician 2013;87:556-66.

3. Elad S, Marshall J, Meyerowitz C, et al. Novel anticoagulants: general overview and practical considerations for dental practitioners. Oral Dis 2016;22:23-32.

4. Costantinides F, Rizzo R, Pascazio L, et al. Managing patients taking novel oral anticoagulants (NOAs) in dentistry: a discussion paper on clinical implications. BMC Oral Health 2016;16:5.

5. Ruff CT, Giugliano RP, Braunwald E, et al. Comparison of the efficacy and safety of new oral anticoagulants with warfarin in patients with atrial fibrillation: a meta-analysis of randomised trials. Lancet 2014;383:955-62.

6. Abraham NS, Singh S, Alexander GC, et al. Comparative risk of gastrointestinal bleeding with dabigatran, rivaroxaban, and warfarin: population based cohort study. BMJ 2015;350:h1857.

7. Healey JS, Eikelboom J, Douketis J, et al. Periprocedural bleeding and thromboembolic events with dabigatran compared with warfarin: results from the Randomized evaluation of Long-Term Anticoagulation therapy (RE-LY) randomized trial. Circulation 2012;126:343-8.

8. Mauprivez C, Khonsari RH, Razouk O, et al. Management of dental extraction in patients undergoing anticoagulant oral direct treatment: a pilot study. Oral Surg Oral Med Oral Pathol Oral Radiol 2016;122:e1 46-e155.

9. Morimoto Y, Yokoe C, Imai Y, et al. Tooth extraction in patients taking nonvitamine K antagonist oral anticoagulants. J Dent Sci 2016;1:11.

10. Pisters R, Lane DA, Nieuwlaat R, et al. A novel user-friendly score (HAS-BLED) to assess 1-year risk of Major bleeding in patients with atrial fibrillation: the Euro Heart Survey. Chest 2010;138:1093-100.

11. Fang $M C$, Go AS, Chang $Y$, et al. A new risk scheme to predict warfarin-associated hemorrhage: the ATRIA (Anticoagulation and risk factors in Atrial Fibrillation) Study. J Am Coll Cardiol 2011;58:395-401.

12. O'Brien EC, Simon DN, Thomas LE, et al. The ORBIT bleeding score: a simple bedside score to assess bleeding risk in atrial fibrillation. Eur Heart J 2015;36:ehv476-64.

13. Bousser MG, Bouthier J, Büller HR, et al. Comparison of idraparinux with vitamin $\mathrm{K}$ antagonists for prevention of thromboembolism in patients with atrial fibrillation: a randomised, open-label, noninferiority trial. Lancet 2008;371:315-21.

14. Senoo K, Proietti M, Lane DA, et al. Evaluation of the HAS-BLED, ATRIA, and ORBIT bleeding risk scores in patients with atrial fibrillation taking warfarin. Am J Med 2016;129:600-7.

15. Japanese society of Oral and Maxillofacial Surgeons, Japanese Society of Gerodontology, Japanese Society of Dentistry for medically compromised patient. guidelines for patients on antithrombotic therapy requiring dental extraction. Tokyo: Gakujutsush Corporation, 2015.

16. Inoue $\mathrm{H}$, Atarashi $\mathrm{H}$, Kamakura S, et al. JCS Joint Working Group. Guidelines for Pharmacotherapy of Atrial Fibrillation (JCS 2013) Digest Version-. Circ J 2014;78:1997-2021.

17. Iwabuchi $\mathrm{H}$, Imai $\mathrm{Y}$, Asanami $\mathrm{S}$, et al. Evaluation of postextraction bleeding incidence to compare patients receiving and not receiving warfarin therapy: a cross-sectional, multicentre, observational study. BMJ Open 2014;4:e005777.
18. DeLong ER, DeLong DM, Clarke-Pearson DL. Comparing the areas under two or more correlated receiver operating characteristic curves: a nonparametric approach. Biometrics 1988;44:837-45.

19. Douketis JD, Spyropoulos AC, Spencer FA, et al. American College of chest Physicians Evidence-Based Clinical Practice guidelines. Chest 2012;14150S:e326S.

20. van Diermen DE, van der Waal I, Hoogstraten J. Management recommendations for invasive dental treatment in patients using oral antithrombotic medication, including novel oral anticoagulants. Oral Surg Oral Med Oral Pathol Oral Radiol 2013;116:709-16.

21. Firriolo FJ, Hupp WS. Beyond warfarin: the new generation of oral anticoagulants and their implications for the management of dental patients. Oral Surg Oral Med Oral Pathol Oral Radiol 2012;113:431-41.

22. Breik O, Tadros R, Devitt P. Thrombin inhibitors: surgical considerations and pharmacology. ANZ J Surg 2013;83:215-21.

23. Hong $\mathrm{CH}$, Islam I. Anti-thrombotic therapy: implications for invasive outpatient procedures in dentistry. J Blood Disorders Transf 2013;4:166

24. Devani P, Lavery KM, Howell CJ. Dental extractions in patients on warfarin: is alteration of anticoagulant regime necessary? $\mathrm{Br} \mathrm{J} \mathrm{Oral}$ Maxillofac Surg 1998;36:107-11.

25. Campbell JH, Alvarado F, Murray RA. Anticoagulation and minor oral surgery: should the anticoagulation regimen be altered? Journal of Oral and Maxillofacial Surgery 2000;58:131-5. discussion 35-6.

26. Blinder D, Manor Y, Martinowitz U, et al. Dental extractions in patients maintained on oral anticoagulant therapy: comparison of INR value with occurrence of postoperative bleeding. Int J Oral Maxillofac Surg 2001;30:518-21.

27. Evans IL, Sayers MS, Gibbons AJ, et al. Can warfarin be continued during dental extraction? results of a randomized controlled trial. $\mathrm{Br} \mathrm{J}$ Oral Maxillofac Surg 2002;40:248-52.

28. Cannon PD, Dharmar VT. Minor oral surgical procedures in patients on oral anticoagulants - a controlled study. Aust Dent J 2003;48:115-8

29. Zanon E, Martinelli F, Bacci C, et al. Safety of dental extraction among consecutive patients on oral anticoagulant treatment managed using a specific dental management protocol. Blood Coagul Fibrinolysis 2003;14:27-30.

30. Ferrieri GB, Castiglioni S, Carmagnola D, et al. Oral surgery in patients on anticoagulant treatment without therapy interruption. $J$ Oral Maxillofac Surg 2007;65:1149-54.

31. Sacco R, Sacco M, Carpenedo M, et al. Oral surgery in patients on oral anticoagulant therapy: a randomized comparison of different intensity targets. Oral Surg Oral Med Oral Pathol Oral Radiol Endod 2007;104:e18-e21.

32. Salam S, Yusuf $\mathrm{H}$, Milosevic A. Bleeding after dental extractions in patients taking warfarin. Br J Oral Maxillofac Surg 2007;45:463-6.

33. Morimoto $\mathrm{Y}$, Niwa H, Minematsu K. Hemostatic management of tooth extractions in patients on oral antithrombotic therapy. $J$ Oral Maxillofac Surg 2008;66:51-7.

34. Pereira CM, Gasparetto PF, Carneiro DS, et al. Tooth extraction in patients on oral anticoagulants: prospective study conducted in 108 Brazilian patients. ISRN Dent 2011;2011:1-4.

35. Kataoka T, Hoshi K, Ando T. Is the HAS-BLED score useful in predicting post-extraction bleeding in patients taking warfarin? A retrospective cohort study. BMJ Open 2016;6:e010471.

36. Green DM, Swets JA. Signal detection Theory and Psychophysics. New York, 1996.

37. Swets JA. Measuring the accuracy of diagnostic systems. Science 1988;240:1285-93.

38. Fischer JE, Bachmann LM, Jaeschke R. A readers' guide to the interpretation of diagnostic test properties: clinical example of Sepsis. Intensive Care Med 2003;29:1043-51.

39. Akobeng AK. Understanding diagnostic tests 2: likelihood ratios, pre- and post-test probabilities and their use in clinical practice. Acta Paediatr 2007;96:487-91.

40. Society JC. Guidelines for management of anticoagulant and antiplatelet therapy in cardiovascular disease. 2009. Available at: http://www.j-circ.or.jp/guideline/pdf/JCS2009_hori_h.pdf (accessed 25 June 2016).

41. Garcia DA, Regan S, Henault LE, et al. Risk of thromboembolism with short-term interruption of warfarin therapy. Arch Intern Med 2008;168:63-9. 